\title{
Mouse syngenic in vitro blood-brain barrier model: a new tool to examine inflammatory events in cerebral endothelium
}

\author{
Caroline Coisne ${ }^{1}$, Lucie Dehouck ${ }^{2}$, Christelle Faveeuw ${ }^{1}$, Yannick Delplace ${ }^{2}$, \\ Florence Miller ${ }^{1}$, Christophe Landry ${ }^{1}$, Céline Morissette ${ }^{3}$, Laurence Fenart ${ }^{2}$, \\ Romeo Cecchelli ${ }^{1}$, Patrick Tremblay ${ }^{3}$ and Bénédicte Dehouck ${ }^{1}$
}

\author{
${ }^{1}$ EA 2465, Unité mixte Université d'Artois, Institut Pasteur de Lille, Lens Cedex, France; ${ }^{2}$ Cellial \\ Technologies, Faculté des Sciences Jean Perrin, rue Jean Souvraz, 62307 Lens, France and ${ }^{3}$ Neurochem Inc., \\ 275, boul. Armand-Frappier, Laval, Québec, Canada H7V 4 A7
}

\begin{abstract}
Although cerebral endothelium disturbance is commonly observed in central nervous system (CNS) inflammatory pathologies, neither the cause of this phenomenon nor the effective participation of bloodbrain barrier (BBB) in such diseases are well established. Observations were mostly made in vivo using mouse models of chronic inflammation. This paper presents a new mouse in vitro model suitable for the study of underlying mechanistic events touching BBB functions during CNS inflammatory disturbances. This model consists of a coculture with both primary cell types isolated from mice. Mouse brain capillary endothelial cell (MBCEC)s coming from brain capillaries are in culture with their in vivo partners and form differentiated monolayers that retain endothelial markers and numerous phenotypic properties of in vivo cerebral endothelium, such as: (1) peripheral distribution of tight junction proteins (occludin, claudin-5, claudin-3 and JAM-1); (2) high trans-endothelium electrical resistance value; (3) attenuated paracellular flux of sucrose and inulin; (4) P-gp expression; (5) no MECA-32 expression. Furthermore, this endothelium expresses cell adhesion molecules described in vivo and shows intracellular cell adhesion molecule-1 and vascular cell adhesion molecule-1 upregulation under lipopolysaccharide-treatment. Therefore, this well-differentiated model using autologous cells appears as a suitable support to reconstitute pathological in vitro BBB model.

Laboratory Investigation (2005) 85, 734-746. doi:10.1038/labinvest.3700281
\end{abstract}

Keywords: BBB; in vitro model; coculture; tight junction; P-glycoprotein; cell adhesion molecule

The maintenance of brain interstitial fluid homeostasis is established by the presence of the bloodbrain barrier (BBB) which can be considered as the main interface between blood and brain parenchymal cells. This endothelium can be distinguished from the other vascular beds by the presence of continuous tight junctions and the absence of fenestration or channel. Both of these characteristics reduce the unspecific transport of molecules across the BBB. The blood/brain exchanges involve specific carrier-mediated transport systems that facilitate the uptake of nutrients. ${ }^{1,2}$

BBB damage commonly associated to inflammatory events has been reported in several central nervous system (CNS) infections and neurodegen-

Correspondence: Dr B Dehouck, PhD, Laboratoire de la BHE, Université d'Artois, Faculté des Sciences, rue Jean Souvraz, SP18, Lens Cedex 62307, France.

E-mail: benedicte.dehouck@univ-artois.fr

Received 22 December 2004; revised 23 February 2005; accepted 23 February 2005 erative diseases. ${ }^{3-5}$ Although BBB permeability disturbances seem to be critical steps as they lead to brain damage, neither the cause of these phenomena nor the effective participation of BBB in such diseases are established.

The cerebral vascular wall was formally considered as a barrier that isolates brain from immunecontrol. However, the presence of immune cells in the brain parenchyma in healthy animals, and their increased cerebral residence in pathogenic conditions, demonstrate that the recruitment of inflammatory cells into the CNS, through the BBB, takes place. $^{6,7}$ In fact, the presence of cell adhesion molecules (CAMs) on the BBB suggests a direct and selective interaction between blood cells and the cerebral endothelium. These molecules, first described in peripheral capillaries, may mediate leukocyte transmigration across the BBB in a multistep process. ${ }^{8,9}$

BBB damages and leukocyte infiltration in brain parenchyma were mostly examined using in vivo mouse models of chronic inflammation, which are 
not adapted to the study of underlying mechanistic events. In vitro approaches have to be undertaken to complete these investigations.

In our laboratory, a coculture of bovine brain capillary endothelial cells (ECs) and rat glial cells has previously been validated as an in vitro BBB model. ${ }^{10}$ However, the use of such bovine/rat system is not suitable for the study of inflammatory events ${ }^{11}$ and the help of a mouse in vitro model would be more appropriate. Unfortunately, most murine models available do not replicate the natural BBB properties observed in vivo. Many reasons can be mentioned to explain these discrepancies: (1) brain ECs come from whichever micro or macrovascular endothelium:12,13 $(2)$ cells used for these models are mostly immortalized;14-16 (3) most cerebral ECs are cultured 'in solo' and thus isolated from their natural in vivo partners known to be of prime importance in inducing differentiation of $\mathrm{BBB}$ properties. ${ }^{17,18}$

We have therefore undertaken the establishment of a mouse BBB model, using primary ECs coming from murine cerebral capillaries cocultivated with murine glial cells. In this model, mouse brain capillary endothelial cells (MBCECs) form differentiated monolayers that retain endothelial markers and numerous in vivo phenotypic BBB properties (tight junction protein and P-glycoprotein (P-gp) expressions), which lead to selective transport through cerebral endothelium.

In order to validate this model for the study of immune events, the expression of CAMs was characterized. Basal expression of CAMs as well as their upregulation by lipopolysaccharide (LPS) confirms that this well-differentiated in vitro model provides a suitable support to reconstitute the pathological BBB conditions, which should facilitate the characterization of the underlying mechanistic events, such as leukocyte migration through the brain endothelium.

\section{Materials and methods}

\section{Chemicals and Antibodies}

$\left[{ }^{3} \mathrm{H}\right]$ inulin $(595 \mathrm{nM}, \quad 0.54 \mathrm{Ci} / \mathrm{mmol}),\left[{ }^{14} \mathrm{C}\right]$ sucrose (75 nM, $600 \mathrm{mCi} / \mathrm{mmol}),\left[{ }^{14} \mathrm{C}\right]$ urea $(1.05 \mu \mathrm{M}, 57 \mathrm{mCi} /$ mmol), $\left[{ }^{3} \mathrm{H}\right]$ vincristine $(50 \mathrm{mM}, \quad 3.3 \mathrm{Ci} / \mathrm{mmol})$, horseradish peroxidase-conjugated sheep antimouse IgG and enhanced chemiluminescence reagent (ECL) were purchased from Amersham Biosciences (Orsay, France). Caffeine $(1 \mu \mathrm{M})$, antipyrine $(1 \mu \mathrm{M})$, cimetidine $(5 \mu \mathrm{M})$ were obtained from SigmaAldrich (St-Quentin Fallavier, France). Mouse fluorescein isothiocyanate (FITC)-conjugated anti$\mathrm{CD} 11 \mathrm{~b}$ and mouse FITC-conjugated IgG2b from Pharmingen (BD Biosciences, Le Pont de Claix, France). Rabbit anti-cow glial fibrillary acidic protein (GFAP), rabbit anti-human von Willebrand factor, mouse anti-human alpha-actin and mouse monoclonal antibody (mAb) anti-P-gp (C219) were obtained from Dako (DakoCytomation S.A., Trappes, France). Rabbit anti-human occludin, rabbit antihuman claudin-5, claudin-3 and claudin-1, from Zymed Laboratories (San Francisco, CA, USA). ICAM-1 (YN1) and OL2 antibodies were generous gift from Professor J Boucraut (Marseille, France) and Dr MS Ghandour (Strasbourg, France) respectively. Rat anti-mouse mAbs for PECAM-1 (MEC 13.3), VCAM-1 (9DB3), ICAM-2 (3C4), MAdCAM-1 (MECA-367), MECA-32, JAM-1 (BV11) and 9B5 as an isotype matched control, were kindly provided by Professor B Engelhardt (Bern, Switzerland). AlexaFluor 488-conjugated goat anti-rat IgG, AlexaFluor 488-conjugated goat anti-rabbit IgG and AlexaFluor 594-conjugated goat anti-rat IgG were purchased from Molecular Probe (Eugene, OR, USA). Cy3-conjugated goat anti-mouse IgG from Jackson Immunoresearch Laboratories (West Grove, PA, USA). Rat anti-mouse CD31 mAb conjugated to phycoerythrine (PE) from Serotec Limited (Cergy Saint-Christophe, France).

\section{Cell Culture Reagents}

The following items were obtained from SigmaAldrich: Dulbecco's modified Eagle's medium (DMEM), HEPES $1 \mathrm{M}$, Amino Acids $50 \times$, Vitamins $100 \times$, tosyl-lysine-chloromethyl-ketone (TLCK), bovin serum albumin (BSA), Hanks balanced salt solution (HBSS) $1 \times$, HBSS Calcium Magnesium $1 \times$, Dextran, LPS (from Escherichia coli, serotype 055: B5). DNase I and collagenase/dispase were purchased from Roche Diagnostics (Meylan, France). Fetal bovine serum (FBS) was obtained from HyClone Laboratories (Logan, UT, USA). Matrigel from BD Biosciences (Le pont Pont de Claix, France). Tissue culture multiwell plates and Transwell $^{\mathrm{TM}}$ polyester membrane (pore size $0.4 \mu \mathrm{m}$ ) inserts were obtained from Costar (Corning Incorporated, NY, USA).

\section{Primary MBCEC Isolation}

\section{Mechanical isolation of brain capillaries from adult cortices}

Cortices, from 4-5 weeks old OF1 mice (Charles River, L'Arbresle, France), were isolated by removing cerebellum, striatum, optic nerves and brain white matter. Outer vessels and meninges were then removed using dry cotton swabs. Preparations were pooled and ground using a Dounce homogenizer (with the large clearance pestle: $0.06-0.08 \mathrm{~mm}$ ) in HBSS $1 \times$ containing $10 \mathrm{mM}$ HEPES and $0.1 \%$ BSA. The resulting homogenate was mixed with $30 \%$ dextran (v/v, molecular weight 100000-200 000) in HBSS $1 \times$ supplemented with $10 \mathrm{mM}$ HEPES and $0.1 \%$ BSA. This suspension was centrifuged at $3000 \mathrm{~g}$ for $25 \mathrm{~min}$ at $4^{\circ} \mathrm{C}$. The neural component and the dextran layer were discarded, the pellet containing the vascular component was resuspended in washing 
buffer (HBSS $1 \times$ supplemented with $10 \mathrm{mM}$ HEPES and $0.1 \%$ BSA). The resulting suspension containing vascular component was filtered through a $59 \mu \mathrm{m}$ nylon mesh, in order to retain large vessels on the mesh surface while the capillaries passed through.

\section{EC collection from isolated capillaries}

The capillary-enriched filtrate was centrifuged at $1000 \mathrm{~g}$ for $7 \mathrm{~min}$ at room temperature. The pellet was then digested in collagenase/dispase $(2 \mathrm{mg} / \mathrm{ml})$ in DMEM supplemented with $10 \mu \mathrm{g} / \mathrm{ml}$ DNase I and $0.147 \mu \mathrm{g} / \mathrm{ml}$ TLCK, for $30 \mathrm{~min}$ at $37^{\circ} \mathrm{C}$ in a shaking water bath.

\section{Seeding of digested brain capillaries}

After several washes, the resulting digested capillary suspension was seeded at 51000 digested capillaries $/ \mathrm{cm}^{2}$ onto tissue culture-treated dishes and/or matrigel-coated inserts (inserts are: Transwell, $0.4 \mu \mathrm{m}, 24 \mathrm{~mm}$ diameter, for flow cytometry, immunoblotting experiments and trans-endothelium electrical resistance (TEER) measurement; Transwell, $0.4 \mu \mathrm{m}, 12 \mathrm{~mm}$ diameter, for permeability studies and immunofluorescence). Culture medium was DMEM supplemented with $20 \%$ FBS, $50 \times$ amino acids, $100 \times$ vitamins, and $50 \mu \mathrm{g} / \mathrm{ml}$ gentamycin. At $24 \mathrm{~h}$ after plating, red blood cells, cell debris and nonadherent cells were removed by washing with medium. Afterwards, the medium was changed every other day and supplemented with $1 \mathrm{ng} / \mathrm{ml}$ basic fibroblast growth factor.

\section{Primary Mouse Glial Cells}

Primary glial cell cultures were prepared from newborn mouse cerebral cortex. After removing the meninges, brain tissue was forced gently through a nylon sieve as described by Booher and Sensenbrenner. ${ }^{19}$ Mixed glial cell cultures were obtained by plating the cells $\left(12500\right.$ cells $\left./ \mathrm{cm}^{2}\right)$, on multi-well plates, in DMEM supplemented with $10 \%$ FBS, $2 \mathrm{mM}$ glutamine and $50 \mu \mathrm{g} / \mathrm{ml}$ gentamycin. The medium was changed twice a week. At 3 weeks after seeding, the cultures were stabilized and used for coculture.

\section{Coculture of MBCECs and Glial Cells}

The day of the MBCEC isolation, resulting digested capillaries were directly plated on the upper side of matrigel-coated inserts. Filters were placed in multiwell dishes containing stabilized glial cell cultures. Under such conditions, ECs migrated from digested capillaries and reached confluence about 4-5 days after plating. Cultures were used 3 days after confluence in order to enhance MBCEC differentiation by glial cells. Coculture medium was the same as the one used for EC growth and was changed every other day.

\section{LPS Exposure}

Confluent cocultured MBCECs were incubated either with LPS in the upper compartment (100 ng/ $\mathrm{ml}$ ) during $17 \mathrm{~h}$ at $37^{\circ} \mathrm{C}$, or with control medium before either processing for immunocytochemistry or flow cytometry analysis.

\section{Immunocytochemical Characterization}

For immunofluorescence experiments performed on fixed cells: fixation with $4 \%$ paraformaldehyde (PFA) in PBS for $10 \mathrm{~min}$, at room temperature, was used for cell surface proteins such as CD11b and OL2. For intracellular proteins, cells were either fixed with $1 \%$ PFA in PBS for 10 min and permeabilized for 1 min with cold acetone (von Willebrand factor and alpha-actin immunostainings), or with $4 \%$ PFA in PBS for $10 \mathrm{~min}$ and permeabilized with $0.1 \%$ Triton X-100 (occludin immunostaining), or methanol/acetone $(\mathrm{v} / \mathrm{v})$ at $-20^{\circ} \mathrm{C}$ for $1 \mathrm{~min}$ (GFAP, claudin-5, claudin-3, claudin-1 and JAM-1 immunostainings). PECAM-1, ICAM-1, VCAM-1, ICAM-2, 9B5 and MAdCAM-1 immunofluorescence experiments were performed on living cells. MBCECs were fixed $10 \mathrm{~min}$ in $1 \%$ PFA, after primary and second antibody reactions. In all cases, nonspecific binding was blocked by incubation with $10 \%$ normal goat serum (NGS) in PBS for 30 min. Primary antibody was diluted in $2 \%$ NGS in PBS and incubated $30 \mathrm{~min}$ at room temperature. After washes in PBS supplemented with $2 \%$ NGS, the secondary antibody was reacted $30 \mathrm{~min}$ at room temperature (AlexaFluor 488 for von Willebrand factor, occludin, claudin-5, claudin-3, claudin-1, JAM-1, ICAM-2, 9B5, GFAP and OL2; AlexaFluor 594 for PECAM-1, ICAM-1,VCAM-1 and 9B5; Cy3 for alpha-actin). Nuclei were stained using Hoechst reagent after secondary antibody labelling. Cells were mounted using Mowiol (Sigma-Aldrich) containing DABCO (Sigma-Aldrich) as an antiquenching agent. Cells were observed with a Leica DMRB fluore scence microscope (Leica Microsystems, Wetzlar, Germany). Images were collected using a Cool SNAP RS Photometrics camera (Leica Microsystems). Images were processed and mounted using Adobe Photoshop software (Adobe Systems, San Jose, CA, USA).

\section{Permeability (Pe) Studies}

Confluent MBCEC monolayer inserts were transferred to 12-well plates containing $1.5 \mathrm{ml}$ of RingerHEPES (150 mM NaCl, $5.2 \mathrm{mM} \mathrm{KCl,} 2.2 \mathrm{mM} \mathrm{CaCl}_{2}$,

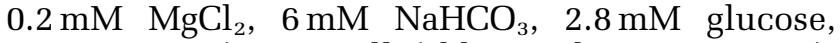
$5 \mathrm{mM}$ HEPES) per well (abluminal compartment). The medium of the apical chambers (luminal compartment) was removed and replaced by $0.5 \mathrm{ml}$ of Ringer-HEPES (RH) containing labelled (inulin, sucrose, urea, vincristine) or unlabelled (caffeine, 
antipyrine, cimetidine) compounds. At different time points, inserts were placed in another well containing $1.5 \mathrm{ml}$ of $\mathrm{RH}$, to minimize an eventual flux of compounds from the abluminal to the luminal compartment. Incubations were performed on a rocking platform at $37^{\circ} \mathrm{C}$. At the end of the experiments, the amount of molecules in the abluminal compartments was measured using a liquid scintillation counter (Perkin Elmer, Courtaboeuf, France) or by HPLC (ThermoFinigan, San Jose, CA, USA).

\section{Pe calculation}

The average volume cleared was plotted vs time and the slope was estimated by linear regression analysis. This slope corresponds to the PSt and PS is the permeability surface area product (in $\mathrm{ml} / \mathrm{min}$ ). The slope of the clearance curve with the control insert (ie, filter coated with matrigel without ECs) was denoted PSf. The PS value for the EC monolayer (PSe) was calculated from: $1 / P S e=1 / P S t-1 / P S f$. The $P S e$ value was divided by the surface area of the filters $\left(1 \mathrm{~cm}^{2}\right.$ for 12 -well Transwell inserts, $12 \mathrm{~mm}$ diameter) to generate the endothelial permeability $(P e$, in $\mathrm{cm} / \mathrm{min})$.

\section{Percentage of passage calculation}

Percentage of passage of each compound through the endothelium was calculated by dividing the percentage of compound going through the MBCEC monolayer insert by the percentage of compound permeability through the control insert. Each percentage corresponds to the amount of solute transport during the whole experiment $(60 \mathrm{~min})$ divided by the initial concentration of the compound. For the correlation between mouse and bovine in vitro models, percentage of endothelial permeability for each solute, obtained using the bovine in vitro $\mathrm{BBB}$ model ${ }^{10}$ was plotted against permeability values obtained using the mouse in vitro model. Data points were used in linear regression analysis to generate the slope and intercept.

\section{Electrical Resistance Measurement}

The TEER of the MBCEC monolayers was measured using a Millicell-ERS apparatus (Millipore, Bedford, MA, USA). The TEER $\left(\Omega \mathrm{cm}^{2}\right)$ of matrigel-coated inserts was measured and subtracted from the resistance value of cocultured ECs to yield the resistance due to the EC monolayers themselves.

\section{Flow Cytometry Analysis}

Confluent MBCEC monolayers in coculture were incubated at $37^{\circ} \mathrm{C}$ with either control medium or $100 \mathrm{ng} / \mathrm{mL}$ LPS for $17 \mathrm{~h}$, added on the upper side of the insert. Afterwards, MBCECs were washed in PBS and trypsinized to carry out flow cytometry analysis. A total of $1 \times 10^{5}-2 \times 10^{5}$ cells were pelleted and resuspended in $50 \mu \mathrm{l}$ of medium (PBS supplemented with $3 \mathrm{mM}$ sodium azide and $2 \%$ FBS) or culture supernatant containing mAb specific for an adhesion molecule. After $30 \mathrm{~min}$ of incubation on ice, cells were washed twice. AlexaFluor 488-conjugated goat anti-rat IgG was added as secondary antibody for another $20 \mathrm{~min}$. After two washes, the binding sites of this second antibody were blocked by addition of PBS $+10 \%$ rat serum before addition of a rat anti-mouse CD31 mAb directly conjugated to PE. The cells were washed twice, resuspended in PBS containing 2\% PFA and analyzed on a FACScalibur flow cytometer using the CellQuest software (Becton Dickinson). Cells were double gated according to (1) the forward and side scatter parameters and (2) the CD31 staining. The number of cells analyzed varied from 3000 to 10000 .

\section{Western Blot Detection of P-gp}

Brain capillaries and trypsinized cells were sonicated in PBS containing a protease inhibitor cocktail (Roche, Mannheim, Germany). Protein concentration was estimated according to the Bradford assay method (Bio-rad Laboratories, Hercules, CA, USA). Samples were resuspended in an equal volume of Laemmli sample buffer containing 20\% mercaptoethanol $(\mathrm{v} / \mathrm{v})$ and incubated at $37^{\circ} \mathrm{C}$. Proteins were separated by $7.5 \%$ SDS- polyacrylamide gel electrophoresis at $100 \mathrm{~V}$ and transferred onto a nitrocellulose membrane by electroblotting overnight at $75 \mathrm{~mA}$ constant current. Nitrocellulose membrane was blocked with PBS, containing calcium (0.9 mM) and magnesium $(0.5 \mathrm{mM})$, supplemented with $5 \%(\mathrm{w} / \mathrm{v})$ milk powder and $0.1 \%(\mathrm{v} / \mathrm{v})$ Tween 20 for at least $1 \mathrm{~h}$. The membrane was probed for $2 \mathrm{~h}$ using mouse monoclonal anti-P-gp antibody (C219; $200 \mathrm{ng} / \mathrm{ml}$ ) at room temperature. After several washes in PBS, secondary horseradish-peroxidase anti-mouse IgG antibody was added for $1 \mathrm{~h}$. Specific protein bands were detected using enhanced chemiluminescence reagent and recorded onto Hyperfilm ECL.

\section{Results}

\section{Assessment of a Mouse In Vitro BBB Model: Coculture of Brain Capillary ECs and Glial Cells}

\section{MBCEC isolation}

Figure 1 describes the MBCEC isolation procedure and shows capillaries and ECs at different steps of the preparation. MBCECs are primary ECs coming from adult mouse cortex capillaries. Firstly, vascular components (Figure 1a) were separated from other brain parenchyma structures. Secondly, capillaries (Figure 1b) were isolated from arterioles and venules. Finally, capillaries were digested (Figure 1c) and seeded on matrigel-coated Petri dishes to follow their dividing process. MBCECs migrated 


\section{Isolation of mouse brain capillary endothelial cells (MBCECs)}

\section{Cortex from}

mice

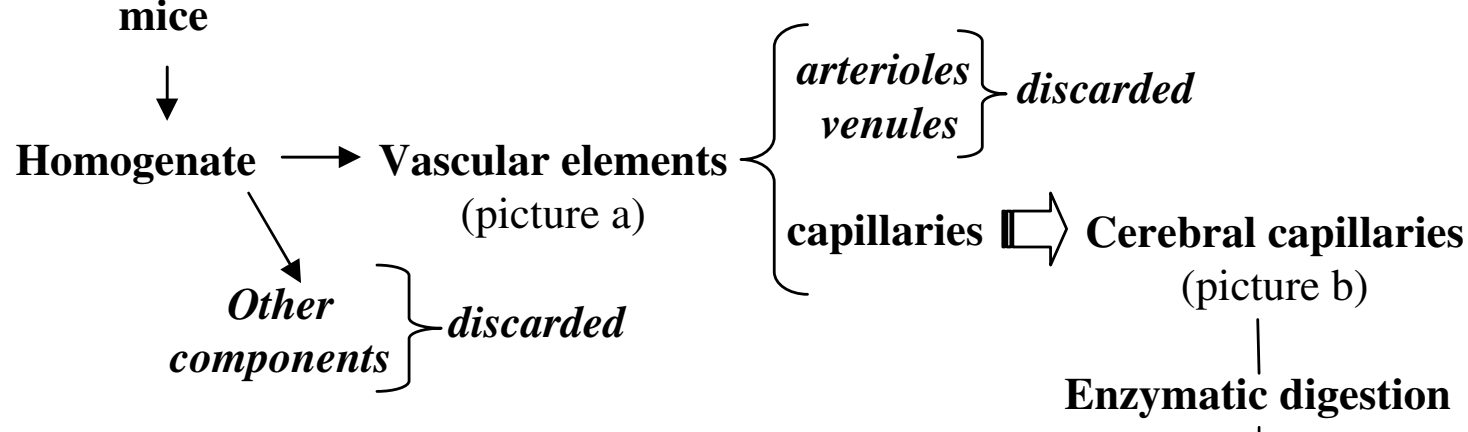

\section{Mouse brain capillary endothelial cells \\ (pictures $\mathrm{d}$ and $\mathrm{e}$ )}

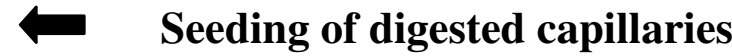
(picture c)
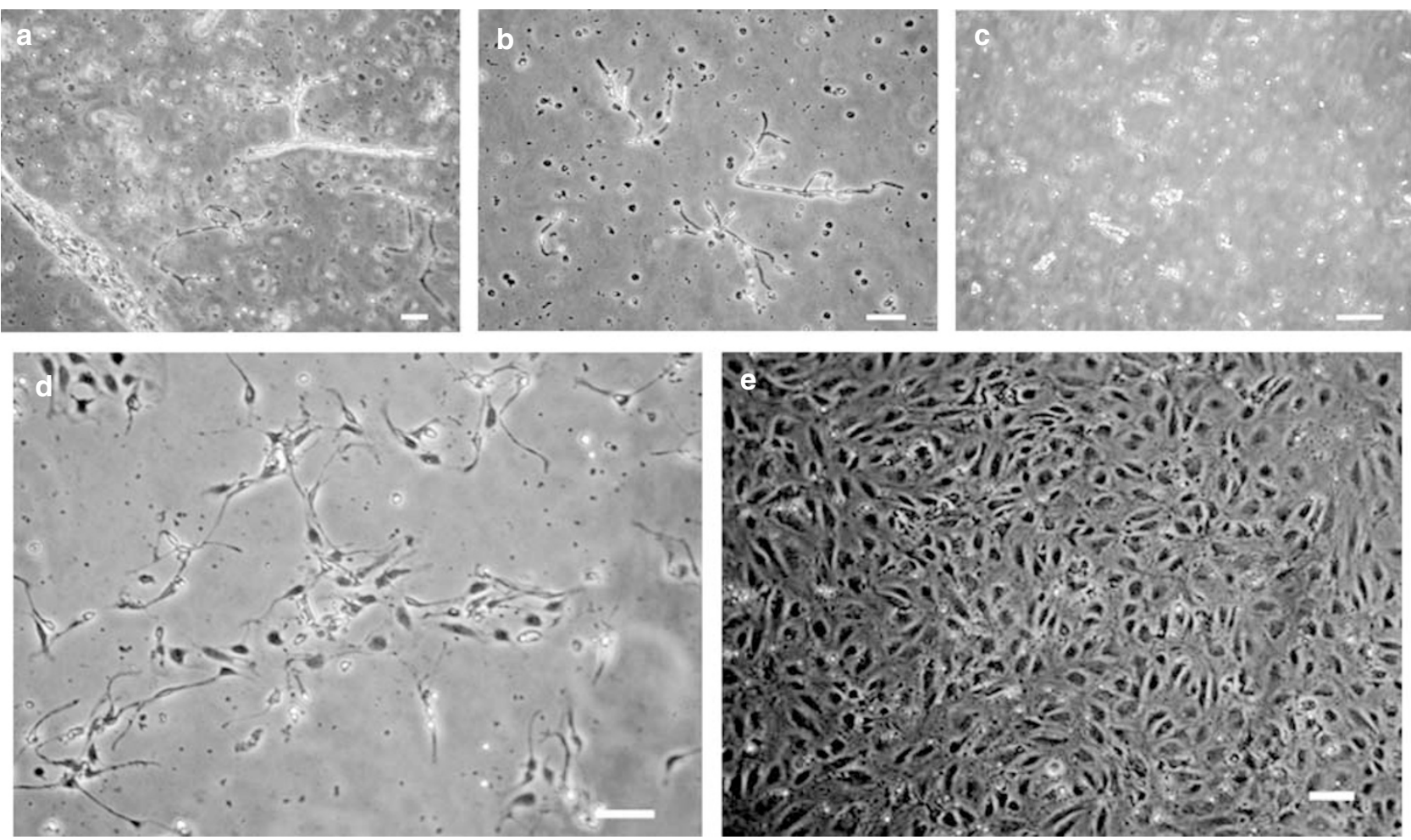

Figure 1 Scheme: Isolation of brain capillary ECs. Pictures: Phase contrast micrographs of (a) vascular components in mouse brain homogenates; (b) cerebral capillaries isolated from vascular components; (c) digested capillary preparation immediately, (d) at $24 \mathrm{~h}$ and (e) 5 days after seeding on matrigel-coated plastic dish (bar $=50 \mu \mathrm{m})$.

from digested capillaries and formed numerous colonies, $24 \mathrm{~h}$ after seeding (Figure 1d). At 5 days after seeding, ECs reached confluence and formed a monolayer (Figure 1e). This experimental procedure attests to the capillary provenance of the ECs and permits their division and growth in culture. 
Glial cells

Glial cell immunolabellings revealed that cultures contain astrocytes but also oligodendrocytes and microglia (Figure 2). The morphology of the cell nuclei following counterstaining of nuclei with Hoechst reagent indicated that the glial population is composed of $84 \%( \pm 2.25)$ astrocytes (Figure $2 b$ ), $10 \%( \pm 3.04)$ oligodendrocytes (Figure $2 \mathrm{c}$ ) and $6 \%$ $( \pm 1.98)$ microglial cells (Figure $2 \mathrm{~d}$ ).

\section{Coculture system}

To assess the coculture system, digested capillaries were directly seeded on matrigel-coated inserts and incubated with primary glial cells as described above. Cells seeded on insert were hardly visible by phase contrast. However, Hoechst staining, performed 5 days after seeding, revealed the confluent MBCEC monolayers on these inserts (Figure 3a). Characterization and experiments were carried out 7 days after setting up the coculture.

\section{MBCEC Characterization in Coculture}

\section{EC markers}

Figure 3 shows intracellular expression of von Willebrand factor (Figure $3 \mathrm{~b}$ ) and cell surface PECAM-1 immunolabelling (Figure 3c). Expression of these EC markers corroborates the formation of complete EC monolayers as expected from a competent vessel wall endothelium. Flow cytometry analysis (FACS) indicated that this confluent monolayer is composed of more than 95\% PECAM-1 positive cells and less than 5\% non-ECs (data not shown). In fact, alpha-actin immunofluorescent staining showed few pericytes in culture (Figure 3d).

\section{$B B B$ characterization}

FACS analysis showed that ECs are negative for MECA-32 (data not shown), a mouse antigen described on nondifferentiated brain ECs and noncerebral ECs, suggesting a good differentiation of the in vitro endothelium. In fact, MBCECs in coculture with glial cells retained many BBB features. Figure $3(\mathrm{e}-\mathrm{h})$ depicts the distribution of tight junction proteins localized at cell borders: claudin-5 (e), occludin (f), claudin-3 (g) and JAM-1 (h). Immunolabelling for claudin-1 did not show any staining (data not shown). TEER measurement gave a high average value of: $777.6 \pm 14.8 \Omega \mathrm{cm}^{2}$.

Another important characteristic of the cerebral endothelium is the expression of the P-gp. A $150 \mathrm{kDa}$ P-gp was detected in mouse brain capillaries by
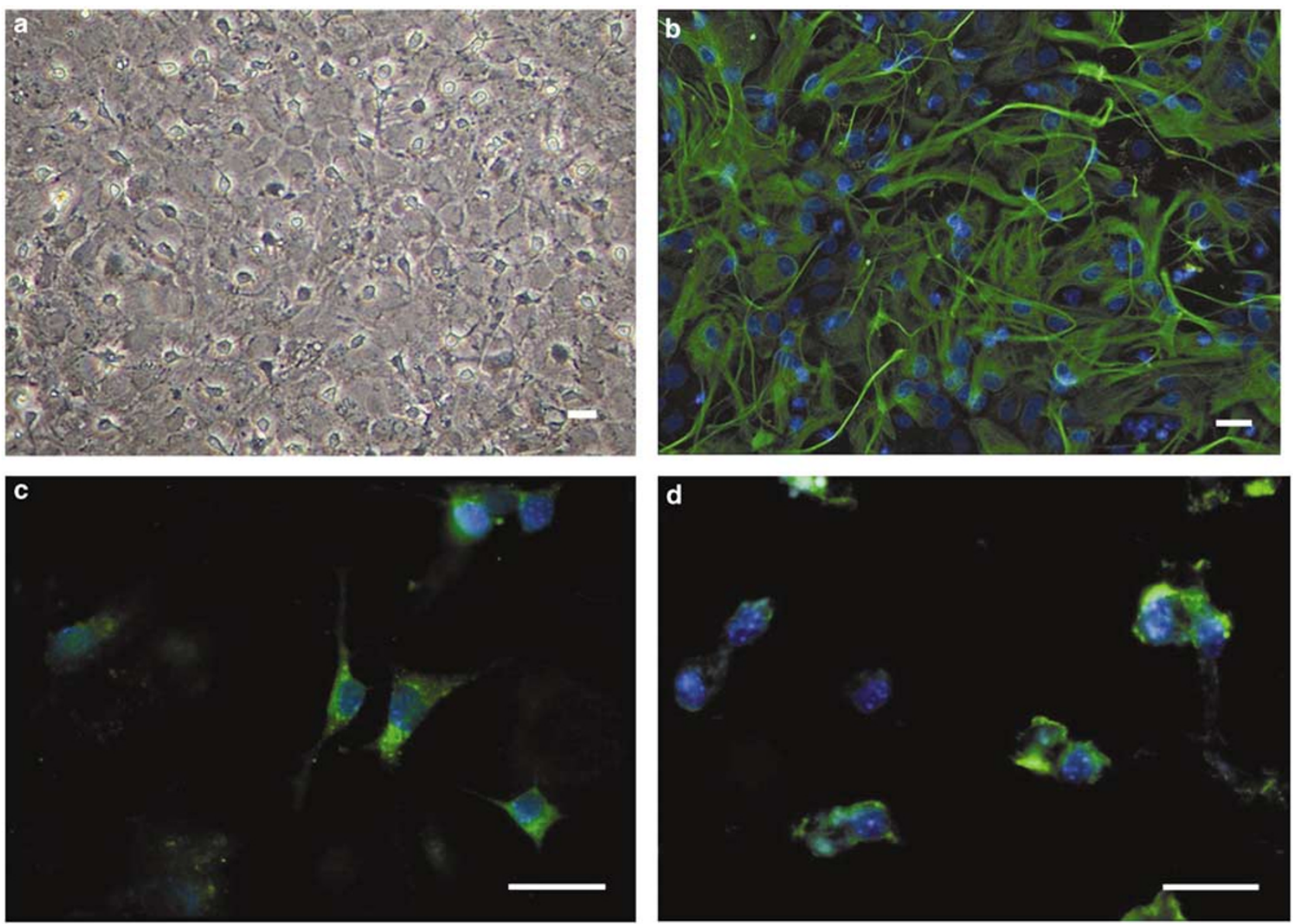

Figure 2 Glial cell culture: (a) phase contrast micrograph of 3-week-old glial cell culture; immunoreactivity is shown for (b) GFAP, (c) OL2, and (d) CD11b (bar $=25 \mu \mathrm{m})$. 

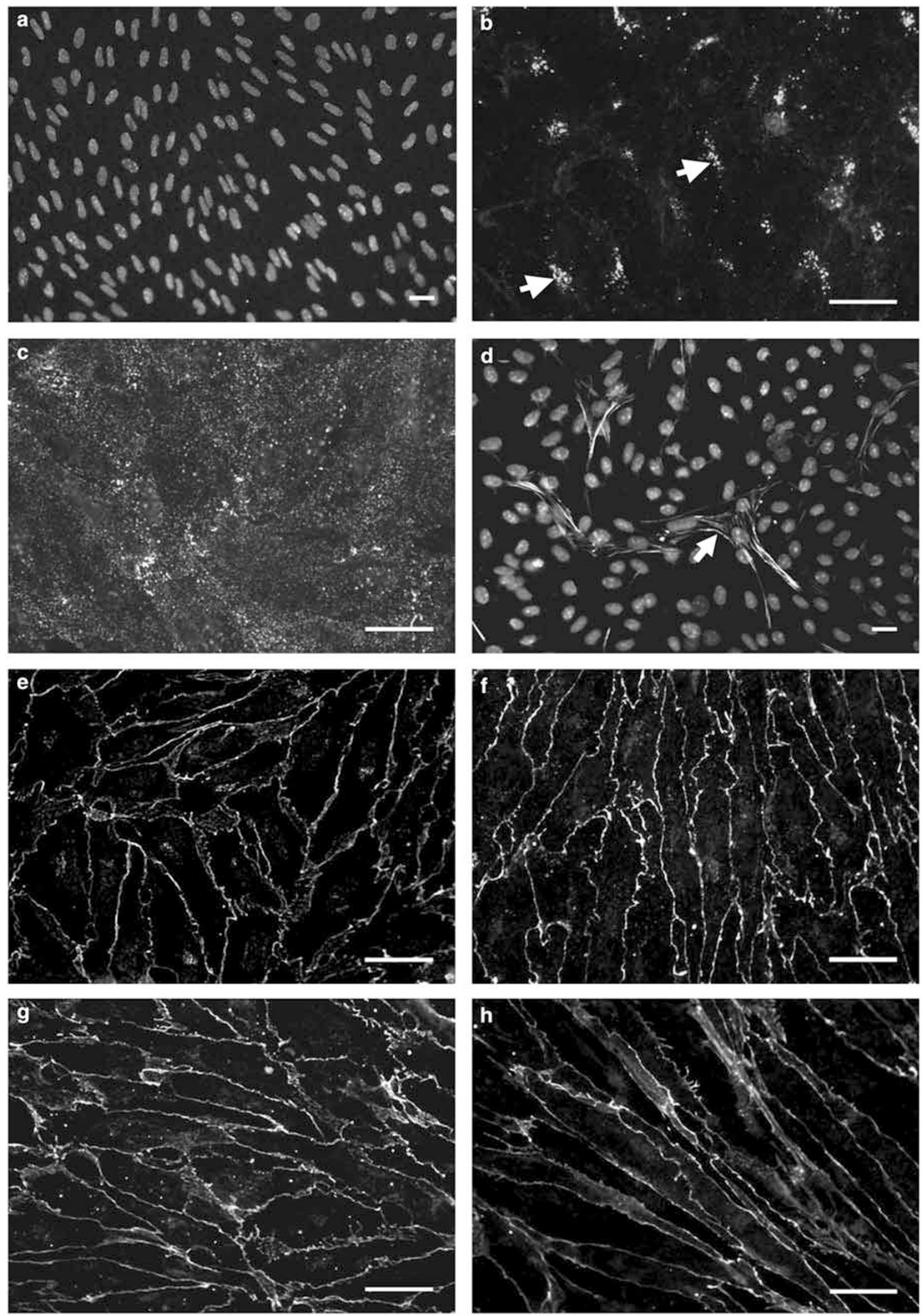

Figure 3 Confluent MBCEC monolayer on matrigel-coated insert and cocultivated with glial cells: (a) nuclear Hoechst staining; (b) Von Willebrand factor immunostaining: perinuclear dotted localization (arrows); (c) PECAM-1 immunostaining: whole cell surface presents dense distribution of immunoreactive dots; (d) nuclear Hoechst staining and alpha-actin staining of pericytes (arrow). Localization of tight junction-associated proteins: (e) Claudin-5, (f) Occludin, (g) Claudin-3, (h) JAM-1 (bar = $25 \mu \mathrm{m})$. 
Western blot technique using C219 antibody (Figure 4a); MBCECs isolated from these capillaries and in coculture with glial cells showed also P-gp expression (Figure 4b). Furthermore, no expression was found in mouse glial cell culture (Figure 4c).

The presence of well-differentiated tight junctions and functional P-gp is confirmed by low permeability rates of small tracers such as sucrose, inulin and vincristine, a P-gp substrate $\left[P e_{\text {sucrose }}=0.27 \pm\right.$ $0.07 \times 10^{-3} \mathrm{~cm} / \mathrm{min}(n=54) ; P e_{\text {inulin }}=0.07 \pm 0.02 \times$ $10^{-3} \mathrm{~cm} / \mathrm{min} \quad(n=13) ; \quad P e_{\text {vincristine }}=0.26 \pm 0.08 \times$ $\left.10^{-3} \mathrm{~cm} / \mathrm{min}(n=9)\right]$. To confirm barrier properties of MBCEC monolayer, permeability rates of different molecules through this endothelium were compared with data obtained using a well-characterized and validated bovine in vitro model. ${ }^{10,20}$ Figure 5 shows that a strong correlation exists between the two in vitro models $\left(R^{2}=0.99\right)$ either for compounds stopped by the cerebral vessel wall such as inulin and cimetidine or for molecules known to easily enter the brain (antipyrine, caffeine).

All the BBB features tested so far attest to the reliability of this in vitro BBB model. Further characterization studies were undertaken to test whether this model is also relevant for studying immune events. As the interaction of blood cells with the cerebral endothelium appears as a critical step modulating neuroinflammation, the vascular expression of adhesion molecules implicated in these interactions was studied.

a

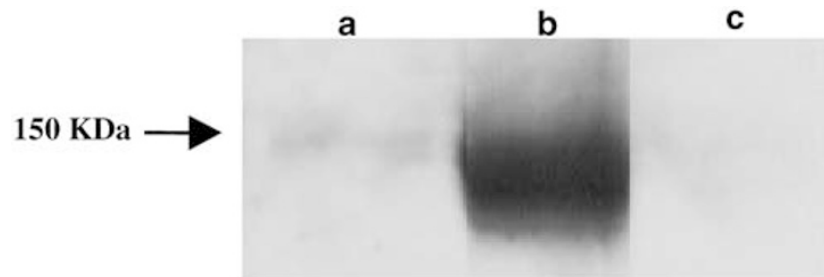

Figure 4 P-gp expression: Western blot using C219 antibody (a) $50 \mu \mathrm{g}$ of mouse brain capillary extract; (b) $25 \mu \mathrm{g}$ proteins from MBCECs cocultivated with glial cells; (c) $50 \mu \mathrm{g}$ proteins from mouse glial cells.

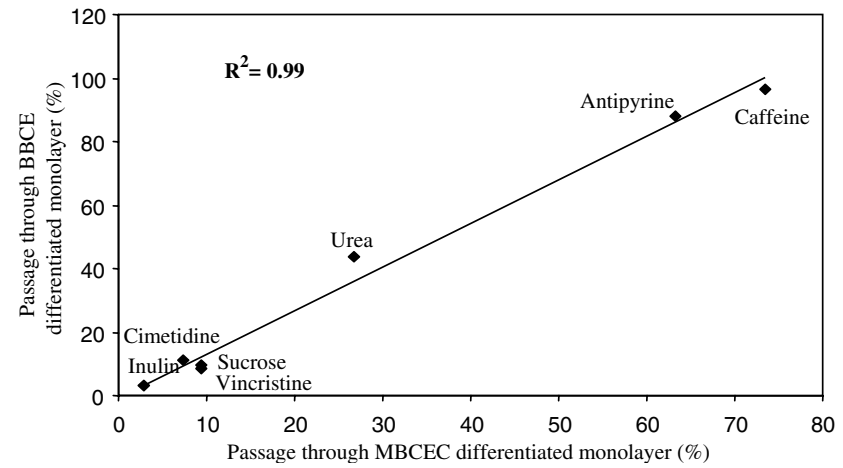

Figure 5 Passage of different compounds through in vitro cerebral-like monolayer: correlation between mouse and bovine in vitro $\mathrm{BBB}$ models. Percent of passage per filter insert through bovine brain capillary endothelial (BBCE) cell monolayers were plotted against values obtained using MBCEC monolayers.
MBCEC expression of adhesion molecules

The expression of adhesion molecules at the cell surface was studied by immunocytochemistry performed directly on live EC monolayers. These stainings were performed for ICAM-1, ICAM-2, VCAM-1, adhesion molecules already described on cerebral endothelium (Figure 6), and for MAdCAM-1 used as negative control (data not shown). Figure 6 shows a basal expression of ICAM-1 (a), ICAM-2 (b) and VCAM-1 (c). Immunostainings of these molecules appeared as distinct dots covering the entire cell surface. Expression of some of these molecules is usually upregulated by inflammatory conditions. Therefore, untreated (Figure 6a-c) and LPS-treated cells $(17 \mathrm{~h}$ at $100 \mathrm{ng} / \mathrm{ml}$ ) (Figure $6 \mathrm{~d}-\mathrm{f}$ ) were compared. ICAM-1 and VCAM-1 immunofluorescent labellings were much more intense on treated cells as revealed by the increased density immunoreactive dots on the LPS-stimulated cells. These results clearly demonstrate an upregulation of these adhesion molecule expressions by LPS exposure. Concerning ICAM-2, the same expression pattern and intensity were observed with or without LPStreatment (Figure 6b vs 6e).

In order to confirm the LPS-dependent upregulation of ICAM-1 and VCAM-1, FACS analyses were performed. Cells were double gated within cell scatter and on positive fluorescence for PECAM-1, to avoid any misinterpretation from the presence of non-ECs (as shown above).

As expected, FACS analysis revealed a basal expression of ICAM-1, ICAM-2, and VCAM-1 at the cell surface while MBCECs were negative for MAdCAM-1 (Figure 7, plain line). Furthermore, a direct quantitative comparison of cell adhesion molecule expression with or without the endotoxin (Figure 7 plain vs bold line) showed (1) an increase in ICAM-1 and VCAM-1 expressions after LPS treatment; (2) no change in ICAM-2 expression; (3) no MAdCAM-1 expression in either condition.

\section{Discussion}

In vitro models are most useful when they closely mimic diverse in vivo situations. According to the literature, establishment of a BBB model implies (1) the provenance of ECs from cerebral capillaries; (2) the use of primary ECs; and (3) the coculture of these ECs with glial cells.

Most murine models available are culture of ECs isolated from brain homogenates. Depending on the technical protocols used for the isolation, these cells are mainly macrovascular or microvascular ECs. ${ }^{12,21,13}$ As a high level of heterogeneity in the properties of EC population has been associated with the diverse locations that they occupy in the vascular tree from which they are isolated, special care must be taken to reduce the diversity in their origin. For instance, Murugesan et $a l^{22}$ suggested that induction of chemokines induced by lyso- 

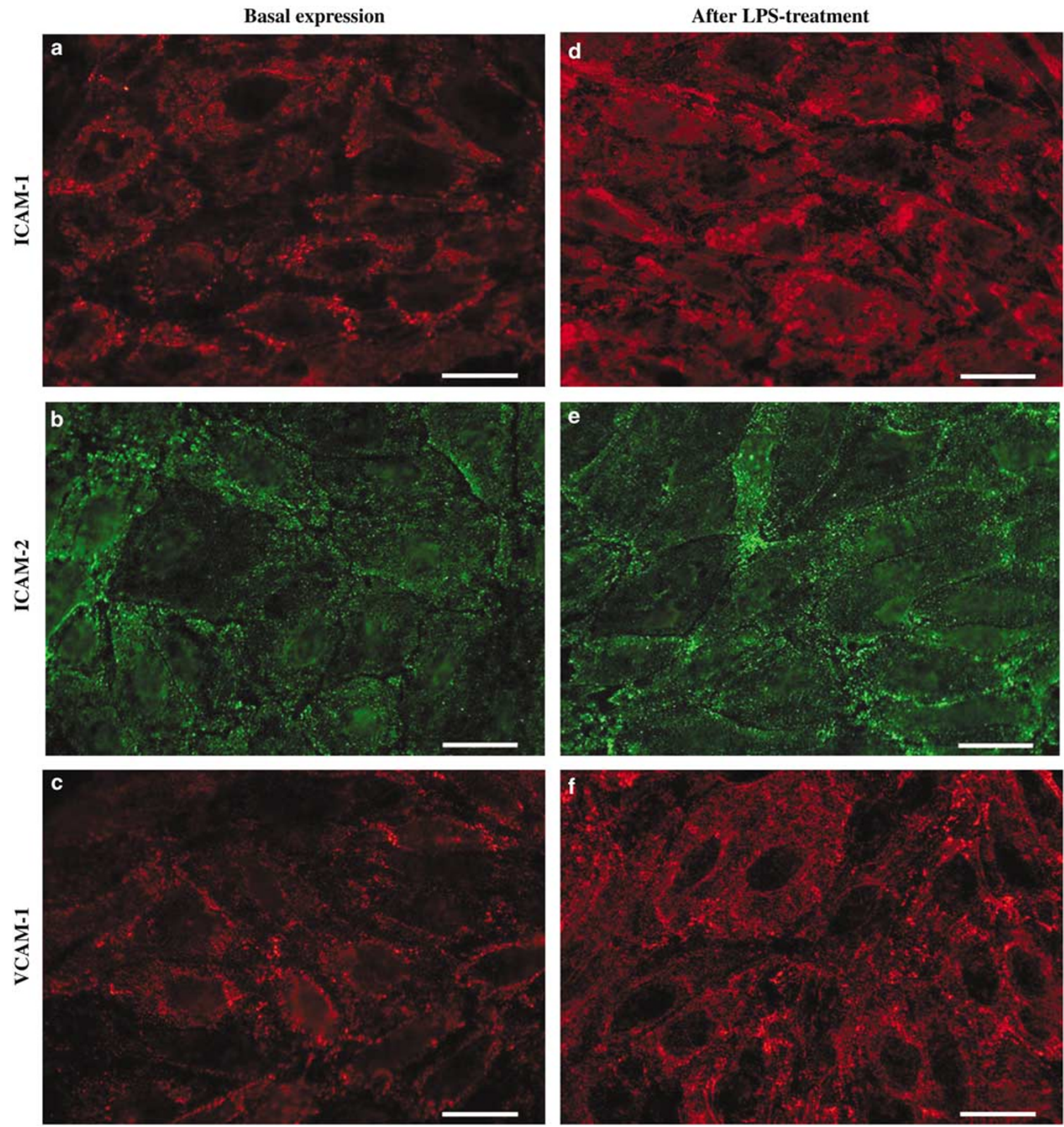

Figure 6 Localization of CAMs on confluent MBCECs seeded on matrigel-coated insert and cocultivated with glial cells. Cell surface expression of ICAM-1, ICAM-2 and VCAM-1 in basal condition (a, b, c respectively) and after endothelium LPS-activation (d, e, f) (bar $=25 \mu \mathrm{m}$ ).

phosphatidylcholine is different in microvascular ECs compared to that of large vessel ECs. In our model, ECs are specifically isolated from cerebral capillaries separated from other vessels in the initial step of the procedure to prevent such heterogeneity.

According to the literature, isolation, viability and proliferation of cerebral ECs appear more difficult when cells are coming from mouse than from bovine and pig species where better outcomes are obtained. This can explain that in most of mouse models ECs need to be transfected to obtain immortalization. Unfortunately, cerebral phenotype can be modified in these conditions. ${ }^{14,23}$ In our procedure, collagenase/dispase digestion of isolated capillaries promoted migration of ECs. Hoechst staining and tight junction immunolabellings showed that MBCECs formed, on matrigel-coated inserts, a complete 

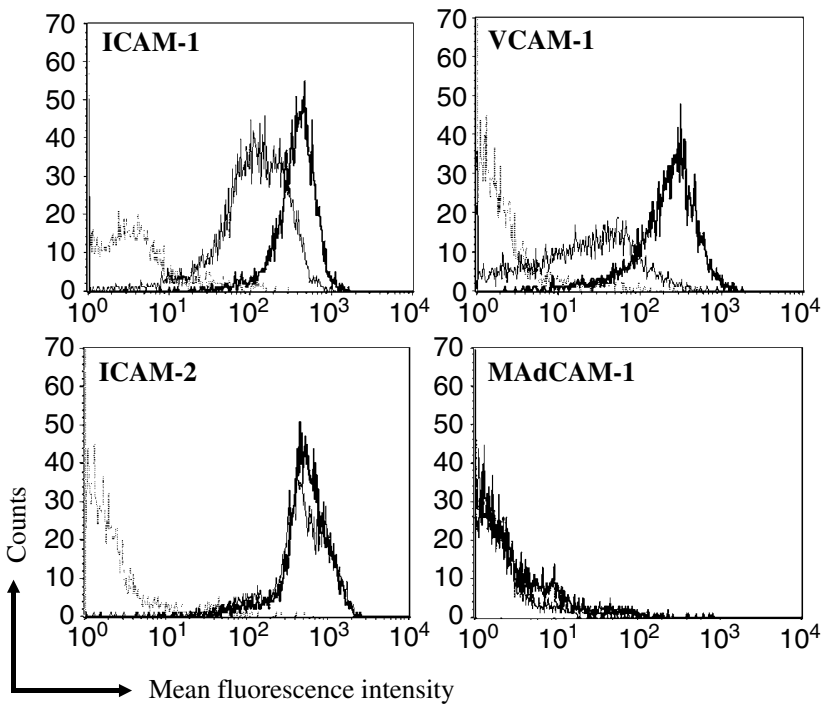

Figure 7 CAM expression on confluent MBCECs seeded on matrigel-coated insert and cocultivated with glial cells: FACS analysis of cells double gated within cell scatter and on positive fluorescence for PECAM-1. Basal expression (normal plain line) and expression after LPS-activation (bold plain line) were compared to each other and to isotype control (dotted line). Isotype control profiles are similar with or without LPS incubation (data not shown).

monolayer of small, tightly packed, nonoverlapping and contact-inhibited cells, as expected for a vessel wall endothelium. The endothelial phenotype was confirmed by the expression of von Willebrand factor and PECAM-1. However, the digestion of capillaries can generate some contaminating pericytes. FACS analysis showed that such cells were scarce compared to PECAM-1 positive cells (5\%vs $95 \%$ ). This low level of contamination was obtained without the use of additional EC purification step such as the use of magnetic beads described by Song and Pachter ${ }^{21}$ and $\mathrm{Wu}$ et al. ${ }^{13}$ Nevertheless, the permeability coefficient of sucrose transport was low in spite of these contaminating cells. Moreover, to avoid any misinterpretations due to the $5 \%$ contaminated cells, FACS analyses were performed on selected PECAM-1-positive cells.

Our procedure allows the isolation of primary mouse capillary ECs, which form, in culture and in few days, an endothelium-like monolayer.

Coculture models have been developed to recreate in vitro natural cooperative cellular interactions between cerebral endothelium and brain parenchyma cells. In fact, both in vivo and in vitro studies suggest that astrocytes modulate the structure and the function of the cerebral endothelium, and consequently the development and maintenance of the BBB. ${ }^{24-27,11}$ Furthermore, in the model described in this paper, ECs are cocultivated with mouse glial cell preparations, which contained not only astrocytes, but also microglia and oligodendrocytes. Recently, Descamps et al ${ }^{11}$ emphasized the importance of cell-cell interactions between glial cell partners. They have shown that purified astrocyte cultures were less efficient than heterogeneous glial cell preparations in the protection of BBB from the deleterious effect induced by LPS. Similarly, Balasingam et $a l^{28}$ have shown that microglia were required for astrocyte reactivity following insults to the neonatal mouse brain. The new mouse in vitro BBB coculture model described here allows cell-cell interactions between natural in vivo partners. BBB property characterizations were performed under these culture conditions.

The lack of MECA-32 expression on MBCECs highlights the differentiated phenotype of this in vitro endothelium as this antigen, expressed on most ECs in adult mouse, is negatively correlated with the differentiation of the cerebral vasculature in vivo. ${ }^{29,30}$ Furthermore, in our coculture system, the expression of occludin, claudin-5, claudin-3 and JAM-1 and peripheral distribution of these proteins suggested the well differentiation of tight junctions. However, claudin-1 was not detected. In vivo, the claudins found in cerebral ECs were first claudin-5 and claudin-1. Discrepancies concerning the presence of either claudin-1 or claudin-3 on cerebral endothelium were due to the use of a crossreactive antibody. Recently, Wolburg et $a l^{31}$ showed the expression of claudin-3 on in vivo healthy mouse CNS vessels. These authors observed the selective loss of claudin-3 immunostaining from tight junctions under pathological conditions and have suggested that this protein was a central component determining the integrity of BBB tight junctions. The presence of claudin-3 in our system validates the well-differentiated nature of this in vitro endothelium. Occludin, claudin-5, claudin-3 and JAM-1 were described as tight junctional transmembrane molecules believed to restrict permeability. ${ }^{32}$ However, studies have shown that peripheral expression of these proteins is not sufficient and not always correlated with the tightness of these structures. ${ }^{33} \mathrm{In}$ our model, TEER values and attenuated flux of sucrose and inulin emphasized the presence and the efficiency of tight junctions on MBCEC monolayers. The expression of P-gp is another important characteristic of BBB. Its expression, generally described in vivo, is often lost in culture conditions. MBCEC monolayer presented expression of P-gp. As a flippase, this protein may be important for the regulation of cell membrane lipids, and therefore contribute to membrane permeability. ${ }^{34}$ In brain ECs, P-gp was also described as a member of the multidrug resistance family, which restricts xenobiotic brain uptake. The expression of this protein is therefore of prime importance to study in vitro drug transport through the BBB. Western blotting showed that P-gp expression is preserved in our culture condition. C219 antibody detected a $150 \mathrm{kDa}$ band. These results are consistent with previous findings, which have shown a $120-155 \mathrm{kDa}$ range for molecular weight of mouse brain P-gp with the heterogeneity arising from the glycosylation state of the protein. ${ }^{35}$ Functional integrity of P-gp on this 
cultured endothelium was confirmed by the low permeability of vincristine, a highly lipophilic molecule known to be P-gp substrate. ${ }^{36}$ On the other hand, glial cells did not express P-gp in this coculture model. This is in agreement with previous findings indicating that astrocytes from rodents express this protein in vitro after stimulation and in vivo under pathological conditions, but rarely under physiological conditions. ${ }^{37-39}$ If tight junctions and P-gp are important to reduce the access of molecules to the brain, other BBB properties such as differential expression of transporters on luminal or basal membranes, can also explain the low permeability of the cerebral endothelium. In our model, the low transport of cimetidine could be explained by the absence of a luminal transporter, as described in vivo on cerebral endothelium. ${ }^{40}$ Furthermore, in spite of BBB properties insuring selective blood/ brain exchanges, cerebral endothelium do not form an absolute barrier and many molecules are known to go readily through the cerebral vessel wall. For instance, agreeing with our in vitro data, multiple reports have shown the effective transport of caffeine and antipyrine in vivo, due to diffusion and presence of transporter. ${ }^{41,42}$

All these results are consistent with permeability data found using an in vitro BBB model validated since $1992 .{ }^{10,20,43}$ In this latter model, developed in our laboratory, bovine brain capillary endothelial (BBCE) cells form a cerebral-like endothelium that is extensively used for evaluating drug transport across the BBB. In fact, Dehouck et $a l,{ }^{10}$ by correlating drug transport between this model and in vivo studies, have demonstrated that bovine in vitro model is suitable to predict and study drug transport. The strong correlation of permeability rate between MBCEC and BBCE cell monolayers now emphasizes the appropriate in vivo-like differentiation of the in vitro mouse endothelium model.

To assess the possible use of this new BBB model for examining immune events, we studied the endothelium expression of adhesion molecules described in the EC/leukocyte interactions.

In this paper, in vitro mouse cerebral endothelium immunostainings showed a constitutive cell surface expression of glycoprotein members of the Ig superfamily of transmembrane proteins: PECAM-1, ICAM-1, ICAM-2 and VCAM-1. These results are consistent with literature-described data that showed in vivo expression of these CAMs on mouse CNS microvessels (PECAM- $1,{ }^{44}$ ICAM- $1,{ }^{45}$ ICAM- ${ }^{46}$ and VCAM-1 ${ }^{47,48}$ ). Expression of these molecules was compared to MAdCAM-1 expression as negative control. MAdCAM-1 has never been shown on cerebral endothelium. ${ }^{49}$ Expression of some of these molecules is usually upregulated during inflammatory conditions. In our hand, immunostainings and FACS analysis on mouse endothelium in vitro culture showed a LPS-dependent upregulation of ICAM-1 and VCAM-1 and no change in ICAM-2 expression. These results agree with in vivo des- cribed data. Authors showed the upregulation of ICAM-1 and VCAM-1 on cerebral endothelium in brain diseases such as EAE, ${ }^{49}$ systemic lupus erythematosus (SLE) ${ }^{45}$ and AIDS neuropathogenesis. $^{50}$ Neither upregulation of ICAM-2 expression ${ }^{46}$ nor de novo synthesis of MAdCAM-1 was described on brain microvascular endothelium. ${ }^{51}$ Thus, adhesion molecule expression, in our mouse in vitro model, correlated with the physiological and pathological in vivo situation. This new model would be suitable for the studies of leukocyte/cerebral EC interactions. Furthermore, this system provides complete accessibility to molecular biology techniques and the preparation of coculture model using knockout mice or experimental disease-induced animal models would be possible to complete in vivo data in the investigation of human pathologic disorders.

\section{Acknowledgements}

This work was supported by grants from the Ministry of Research of France (fellowship to C Coisne) and by the 'Conseil Régional du NordPas-de-Calais' (fellowship to F Miller). We are very grateful to Professor B Engelhardt (Theodor Kocher Institute, University of Bern, Switzerland), Professor J Boucraut (Laboratoire NICN UMR 6184, Faculté de Médecine Timone, Marseille) and Dr MS Ghandour (Centre National de la Recherche Scientifique, Strasbourg, France) to their generous gifts of antibodies. We thank Mrs Nadine Tremblay for technical assistance and Dr Valérie Buée for critical reading of the manuscript.

\section{References}

1 Dehouck B, Fenart L, Dehouck MP, et al. A new function for the LDL receptor: transcytosis of LDL across the blood-brain barrier. J Cell Biol 1997;138: 877-889.

2 Descamps L, Dehouck MP, Torpier G, et al. Receptormediated transcytosis of transferrin through bloodbrain barrier endothelial cells. Am J Physiol 1996;270: H1149-H1158.

3 Tunkel AR, Scheld WM. Pathogenesis and pathophysiology of bacterial meningitis. Clin Microbiol Rev 1993;6:118-136.

4 Leib SL, Tauber MG. Pathogenesis of bacterial meningitis. Infect Dis Clin North Am 1999;13:527-548.

5 Kraus J, Ling AK, Hamm S, et al. Interferon-beta stabilizes barrier characteristics of brain endothelial cells in vitro. Ann Neurol 2004;56:192-205.

6 Hickey WF, Hsu BL, Kimura H. T-lymphocyte entry into the central nervous system. J Neurosci Res 1991; 28:254-260.

7 Tsukada N, Matsuda M, Miyagi K, et al. Cytotoxicity of $\mathrm{T}$ cells for cerebral endothelium in multiple sclerosis. J Neurol Sci 1993;117:140-147. 
8 Butcher EC. Leukocyte-endothelial cell recognition: three (or more) steps to specificity and diversity. Cell 1991;67:1033-1036.

9 Springer TA. Traffic signals on endothelium for lymphocyte recirculation and leukocyte emigration. Annu Rev Physiol 1995;57:827-872.

10 Dehouck MP, Jolliet Riant P, Bree F, et al. Drug transfer across the blood-brain barrier: correlation between in vitro and in vivo models. J Neurochem 1992;58: 1790-1797.

11 Descamps L, Coisne C, Dehouck B, et al. Protective effect of glial cells against lipopolysaccharidemediated blood-brain barrier injury. Glia 2003;42: 46-58.

12 Song L, Pachter JS. Monocyte chemoattractant protein1 alters expression of tight junction-associated proteins in brain microvascular endothelial cells. Microvasc Res 2004;67:78-89.

$13 \mathrm{Wu}$ Z, Hofman FM, Zlokovic BV. A simple method for isolation and characterization of mouse brain microvascular endothelial cells. J Neurosci Methods 2003; 130:53-63.

14 Röhnelt RK, Hoch G, Reiss Y, et al. Immunosurveillance modelled in vitro: naive and memory $\mathrm{T}$ cells spontaneously migrate across unstimulated microvascular endothelium. Int Immunol 1997;9:435-450.

15 Roux F, Durieu Trautmann O, Chaverot N, et al. Regulation of gamma-glutamyl transpeptidase and alkaline phosphatase activities in immortalized rat brain microvessel endothelial cells. J Cell Physiol 1994;159:101-113.

16 Greenwood J, Pryce G, Devine L, et al. SV40 large T immortalised cell lines of the rat blood-brain and blood-retinal barriers retain their phenotypic and immunological characteristics. J Neuroimmunol 1996; 71:51-63.

17 Debault LE, Cancilla PA. Gamma-glutamyl transpeptidase in isolated brain endothelial cells: induction by glial cells in vitro. Science 1980;207:653-655.

18 Dehouck MP, Meresse S, Delorme P, et al. An easier, reproducible, and mass-production method to study the blood-brain barrier in vitro. J Neurochem 1990; 54:1798-1801.

19 Booher J, Sensenbrenner M. Growth and cultivation of dissociated neurons and glial cells from embryonic chick, rat and human brain in flask cultures. Neurobiology 1972;2:97-105.

20 Cecchelli R, Dehouck B, Descamps L, et al. In vitro model for evaluating drug transport across the blood-brain barrier. Adv Drug Deliv Rev 1999;36: 165-178.

21 Song L, Pachter JS. Culture of murine brain microvascular endothelial cells that maintain expression and cytoskeletal association of tight junction-associated proteins. In Vitro Cell Dev Biol Anim 2003;39:313-320.

22 Murugesan G, Sandhya Rani MR, Gerber CE, et al. Lysophosphatidylcholine regulates human microvascular endothelial cell expression of chemokines. J Mol Cell Cardiol 2003;35:1375-1384.

23 Omidi Y, Campbell L, Barar J, et al. Evaluation of the immortalised mouse brain capillary endothelial cell line, b.End3, as an in vitro blood-brain barrier model for drug uptake and transport studies. Brain Res 2003;990:95-112.

24 Risau W, Hallmann R, Albrecht U, et al. Brain induces the expression of an early cell surface marker for blood-brain barrier-specific endothelium. EMBO J 1986;5:3179-3183.

25 Janzer RC, Raff MC. Astrocytes induce blood-brain barrier properties in endothelial cells. Nature 1987; 325:253-257.

26 Raub TJ, Kuentzel SL, Sawada GA. Permeability of bovine brain microvessel endothelial cells in vitro: barrier tightening by a factor released from astroglioma cells. Exp Cell Res 1992;199:330-340.

27 Dehouck B, Dehouck MP, Fruchart JC, et al. Upregulation of the low density lipoprotein receptor at the blood-brain barrier: intercommunications between brain capillary endothelial cells and astrocytes. J Cell Biol 1994;126:465-473.

28 Balasingam V, Dickson K, Brade A, et al. Astrocyte reactivity in neonatal mice: apparent dependence on the presence of reactive microglia/macrophages. Glia 1996;18:11-26.

29 Hallmann R, Mayer DN, Berg EL, et al. Novel mouse endothelial cell surface marker is suppressed during differentiation of the blood-brain barrier. Dev Dyn 1995;202:325-332.

30 Engelhardt B, Conley FK, Butcher EC. Cell adhesion molecules on vessels during inflammation in the mouse central nervous system. J Neuroimmunol 1994; 51:199-208.

31 Wolburg H, Wolburg Buchholz K, Kraus J, et al. Localization of claudin-3 in tight junctions of the blood-brain barrier is selectively lost during experimental autoimmune encephalomyelitis and human glioblastoma multiforme. Acta Neuropathol (Berl) 2003; 105:586-592.

32 Wolburg H, Lippoldt A. Tight junctions of the bloodbrain barrier: development, composition and regulation. Vasc Pharmacol 2002;38:323-337.

33 Hamm S, Dehouck B, Kraus J, et al. Astrocyte mediated modulation of blood-brain barrier permeability does not correlate with a loss of tight junction proteins from the cellular contacts. Cell Tissue Res 2004;315: 157-166.

34 Higgins CF. Flip-flop: the transmembrane translocation of lipids. Cell 1994;79:393-395.

35 Jette L, Pouliot JF, Murphy GF, et al. Isoform I (mdr3) is the major form of P-glycoprotein expressed in mouse brain capillaries. Evidence for cross-reactivity of antibody C219 with an unrelated protein. Biochem J 1995;305:761-766

36 Fenart L, Buee Scherrer V, Descamps L, et al. Inhibition of P-glycoprotein: rapid assessment of its implication in blood-brain barrier integrity and drug transport to the brain by an in vitro model of the blood-brain barrier. Pharm Res 1998;15:993-1000.

37 Zhang L, Ong WY, Lee T. Induction of P-glycoprotein expression in astrocytes following intracerebroventricular kainate injections. Exp Brain Res 1999;126 509-516.

38 Monville C, Fages C, Feyens AM, et al. Astroglial expression of the P-glycoprotein is controlled by intracellular CNTF. BMC Cell Biol 2002;3:20.

39 Schlachetzki F, Pardridge WM. P-glycoprotein and caveolin-1alpha in endothelium and astrocytes of primate brain. Neuroreport 2003;14:2041-2046.

40 Mori S, Takanaga H, Ohtsuki S, et al. Rat organic anion transporter 3 (rOAT3) is responsible for brain-to-blood efflux of homovanillic acid at the abluminal membrane of brain capillary endothelial cells. J Cereb Blood Flow Metab 2003;23:432-440. 
41 McCall AL, Millington WR, Wurtman RJ. Blood-brain barrier transport of caffeine: dose-related restriction of adenine transport. Life Sci 1982;31:2709-2715.

42 Eckman WW, Phair RD, Fenstermacher JD, et al. Permeability limitation in estimation of local brain blood flow with $\left[{ }^{14} \mathrm{C}\right]$ antipyrine. Am J Physiol 1975; 229:215-221.

43 Berezowski V, Landry C, Lundquist S, et al. Transport screening of drug cocktails through an in vitro bloodbrain barrier: is it a good strategy for increasing the throughput of the discovery pipeline? Pharm Res 2004;21:756-760.

44 Graesser D, Solowiej A, Bruckner M, et al. Altered vascular permeability and early onset of experimental autoimmune encephalomyelitis in PECAM-1-deficient mice. J Clin Invest 2002;109:383-392.

45 Zameer A, Hoffman SA. Increased ICAM-1 and VCAM1 expression in the brains of autoimmune mice. J Neuroimmunol 2003;142:67-74.

46 Fabry Z, Waldschmidt MM, Hendrickson D, et al. Adhesion molecules on murine brain microvascular endothelial cells: expression and regulation of ICAM-1 and Lgp 55. J Neuroimmunol 1992;36:1-11.
47 Vajkoczy P, Laschinger M, Engelhardt B. Alpha4integrin-VCAM-1 binding mediates G protein-independent capture of encephalitogenic $\mathrm{T}$ cell blasts to CNS white matter microvessels. J Clin Invest 2001;108: 557-565.

48 Engelhardt B, Laschinger M, Schulz M, et al. The development of experimental autoimmune encephalomyelitis in the mouse requires alpha4-integrin but not alpha4beta7-integrin. J Clin Invest 1998;102: 2096-2105.

49 Steffen BJ, Butcher EC, Engelhardt B. Evidence for involvement of ICAM-1 and VCAM-1 in lymphocyte interaction with endothelium in experimental autoimmune encephalomyelitis in the central nervous system in the SJL/J mouse. Am J Pathol 1994;145: 189-201.

50 MacLean AG, Orandle MS, MacKey J, et al. Characterization of an in vitro rhesus macaque blood-brain barrier. J Neuroimmunol 2002;131:98-103.

51 Steffen BJ, Breier G, Butcher EC, et al. ICAM-1, VCAM-1, and MAdCAM-1 are expressed on choroid plexus epithelium but not endothelium and mediate binding of lymphocytes in vitro. Am J Pathol 1996;148:1819-1838. 